\title{
Das präventive und therapeutische Potenzial vegetarischer und veganer
}

\section{Ernährung}

\section{Aै}

Vegetarische Ernährungsformen verringern das

Risiko für zahlreiche ernährungsassoziierte chronische

Erkrankungen und bieten bisher kaum genutzte

therapeutische Möglichkeiten - Ein Überblick über

den aktuellen Stand der Wissenschaft

Markus Keller

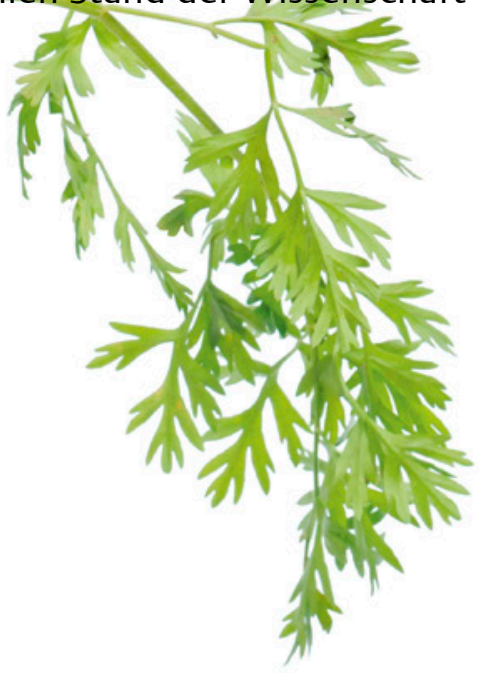

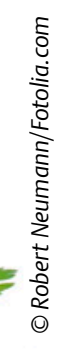

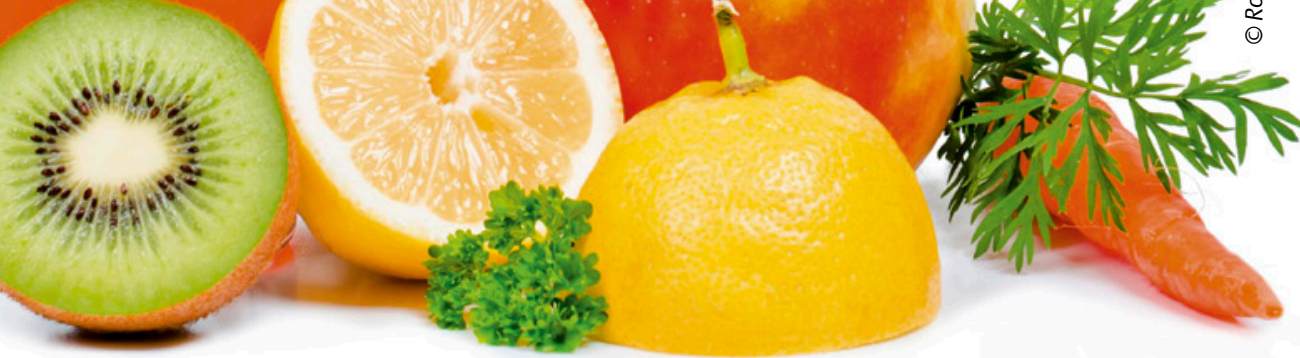

\section{Zusammenfassung}

Zahlreiche Studien zeigen, dass Vegetarier und Veganer ein deutlich verringertes Risiko für ernährungsassoziierte Erkrankungen, wie Übergewicht, Typ-2-Diabetes, Hypertonie, kardiovaskuläre Erkrankungen und bestimmte Krebsarten, aufweisen. Mit den meisten Nährstoffen sind Vegetarier und Veganer gut bis sehr gut versorgt, auf die Zufuhr potenziell kritischer Nährstoffe muss jedoch geachtet werden. Vegetarische Kostformen haben ein großes, bisher kaum genutztes Potenzial zur Prävention und Therapie der genannten Erkrankungen sowie zur Gesundheitsförderung.

Nach Schätzungen des Bundesministeriums für Gesundheit verursachen ernährungsmitbedingte chronische Erkrankungen etwa $30 \%$ aller Krankheitskosten in Deutschland - dies entsprach etwa $88 \mathrm{Mrd}$. Euro im Jahr 2011 (hochgerechnet nach [1, 2]). Zahlreiche wissenschaftliche Studien zeigen, dass Vegetarier und Veganer ein deutlich verringertes Risiko für diese Erkrankungen aufweisen [3]. Diese Befunde gelten unabhängig von der insgesamt gesünderen Lebensweise. Zwar muss bei vegetarischer und veganer Ernährung auf die ausreichende $\mathrm{Zu}$ fuhr bestimmter Nährstoffe geachtet werden. Mit vielen Nährstoffen sind Vegetarier und Veganer jedoch genauso gut oder sogar besser versorgt als Fleischesser. In diesem Übersichtsartikel wird der aktuelle Stand der Wissenschaft zur Krankheitsprävention sowie zur Nährstoffversorgung bei vegetarischen Ernährungsformen dargestellt.

\section{Studien mit Vegetariern}

Gesicherte Informationen zum unterschiedlichen Erkrankungs- und Sterberisiko von Vegetariern und Nicht-Vegetariern stammen aus prospektiven Kohortenstudien. Dabei werden die ermittelten Ergebnisse für zahl- 
reiche Störfaktoren (confounder) adjustiert, um beobachtete Zusammenhänge soweit wie möglich auf die unterschiedliche Ernährungsweise der Untersuchungsgruppen zurückführen zu können. Wichtige Störfaktoren in ernährungsepidemiologischen Studien sind Körpergewicht bzw. BMI, Tabak- und Alkoholkonsum sowie körperliche Aktivität. Aktuelle Daten liefern die derzeit laufende EPIC-Oxford-Studie (über 65000 Teilnehmer) sowie die Adventist Health Study 2 (über 96000 Teilnehmer). Einen Überblick über die bisher durchgeführten Kohortenstudien mit Vegetariern gibt Tab. 1.

\section{Prävention \\ Übergewicht/Adipositas}

Körpergewicht und Body-Mass-Index (BMI) von Vegetariern und Veganern liegen im Durchschnitt niedriger als bei Mischköstlern. In der Adventist Health Study 2 steigt der BMI von Veganern über Lakto-Ovo-Vegetarier und Fischesser hin zu Fleischessern kontinuierlich an [4]. Nach Ansicht der Auto- ren der Studie weist der Unterschied von 5 BMI-Einheiten zwischen Veganern und Fleischessern auf ein erhebliches Potenzial des Vegetarismus hin, vor Übergewicht zu schützen. Entsprechend ist auch der Anteil Übergewichtiger bei Vegetariern deutlich geringer als bei Nicht-Vegetariern [3].

Verantwortlich dafür ist v. a. der niedrigere Energiegehalt pflanzlicher Kost. Sie enthält mehr komplexe Kohlenhydrate und Ballaststoffe sowie weniger Protein und Gesamtfett und liefert dadurch bei gleichem Volumen weniger Energie als Mischkost. Die höhere Ballaststoffzufuhr sorgt zusätzlich für eine schnellere und länger anhaltende Sättigung. Vegetarische Ernährungsformen können somit aufgrund ihrer hohen Nährstoff- und niedrigen Energiedichte zur Gewichtsregulation bzw. für den Erhalt eines gesunden Körpergewichts empfohlen werden [5].

\section{Diabetes mellitus Typ 2}

Vegetarier erkranken deutlich seltener an Typ-2-Diabetes als Nicht-Vegetarier. In ei- ner Auswertung der Adventist Health Study 2 ( $n=41387$ ) lag das Risiko, erstmalig an Diabetes mellitus (Typ 1 und 2) zu erkranken, bei Lakto-Ovo-Vegetariern um etwa $38 \%$ und bei Veganern um etwa $62 \%$ niedriger als bei Fleischessern (jeweils nach Adjustierung für BMI und zahlreiche weitere Störfaktoren) [6]. Auch weitere Studien zeigen für Vegetarier und Veganer ein niedrigeres Diabetesrisiko [3].

Als Hauptursache für diese Befunde gelten das geringere Körpergewicht und die höhere Ballaststoffzufuhr bei pflanzenbasierter Ernährung. Ballaststoffe sowie die bei Vegetariern und Veganern übliche geringere Zufuhr von Gesamtfett, insbesondere von gesättigten Fettsäuren, wirken der Insulinresistenz entgegen. Vegetarier weisen auch bei gleichem Körpergewicht wie Nicht-Vegetarier niedrigere Blutglukose- und Insulinwerte im Nüchternblut sowie eine höhere Insulinsensitivität auf [7]. Mit steigendem Fleischverzehr erhöht sich - unabhängig von weiteren Ernährungsfaktoren - das Diabetesri-

\section{Prospektive Kohortenstudien mit Vegetariern}

\begin{tabular}{|c|c|c|c|c|c|}
\hline Studie & Land & $\begin{array}{l}\text { Beginn } \\
\text { [Jahr] }\end{array}$ & $\begin{array}{l}\text { Letzter Follow- } \\
\text { up [Jahr] }\end{array}$ & $\begin{array}{l}\text { Untersuchte Gruppen } \\
\text { (Anzahl der Teilnehmer, gerundet) }\end{array}$ & $\begin{array}{l}\text { (Haupt)Untersuchungsziel: } \\
\text { Zusammenhänge zwischen } \\
\text { Ernährungsmuster und ... }\end{array}$ \\
\hline Adventist Mortality Study & USA & 1960 & 1986 & $\begin{array}{l}\text { Lakto-Ovo-Vegetarier (7920) } \\
\text { Nicht-Vegetarier (6960) }\end{array}$ & Sterblichkeit \\
\hline Adventist Health Study & USA & 1976 & 1988 & $\begin{array}{l}\text { Lakto-Ovo-Vegetarier (7190) } \\
\text { Nicht-Vegetarier (7460) }\end{array}$ & $\begin{array}{l}\text { Häufigkeit chronischer Erkrankungen, } \\
\text { Sterblichkeit }\end{array}$ \\
\hline $\begin{array}{l}\text { Vegetarierstudie des } \\
\text { DKFZ* }\end{array}$ & Deutschland & 1978 & 1999 & $\begin{array}{l}\text { Lakto-Ovo-Vegetarier (1170) } \\
\text { Veganer }(60) \\
\text { Gesundheitsbewusste Nicht-Vegetarier (680) }\end{array}$ & Sterblichkeit \\
\hline Oxford Vegetarian Study & Großbritannien & 1980 & 2000 & $\begin{array}{l}\text { Vegetarier** }^{* *}(4670) \\
\text { Nicht-Vegetarier }(6370)\end{array}$ & $\begin{array}{l}\text { Sterblichkeit, Häufigkeit chronischer } \\
\text { Erkrankungen }\end{array}$ \\
\hline EPIC $* * * *$-Oxford & Großbritannien & 1993 & $\begin{array}{l}2007 \text { wird } \\
\text { fortgeführt }\end{array}$ & $\begin{array}{l}\text { Fleischesser }(33880) \\
\text { Fischesser }(10110) \\
\text { Lakto-Ovo-Vegetarier }(18840) \\
\text { Veganer }(2600)\end{array}$ & $\begin{array}{l}\text { Häufigkeit chronischer Erkrankungen, } \\
\text { Sterblichkeit, Nährstoffzufuhr }\end{array}$ \\
\hline UK Womens' Cohort Study & Großbritannien & 1995 & $\begin{array}{l}2004 \text { wird } \\
\text { fortgeführt }\end{array}$ & $\begin{array}{l}\text { Fleischesserinnen }(24740) \\
\text { Fischesserinnen }(4160) \\
\text { Vegetarierinnen }{ }^{* * *}(6480)\end{array}$ & Krebshäufigkeit, Nährstoffzufuhr \\
\hline Adventist Health Study 2 & USA und Kanada & 2002 & $\begin{array}{l}\text { - wird } \\
\text { fortgeführt }\end{array}$ & $\begin{array}{l}\text { Fleischesser }(45200) \\
\text { Selten Fleischesser }(5900) \\
\text { Fischesser }(11000) \\
\text { Lakto-Ovo-Vegetarier }(30500) \\
\text { Veganer }(4190)\end{array}$ & $\begin{array}{l}\text { Häufigkeit chronischer Erkrankungen, } \\
\text { Sterblichkeit (insbesondere Krebs) }\end{array}$ \\
\hline
\end{tabular}

Tab. 1 Prospektive Kohortenstudien mit Vegetariern. Aktualisiert nach [45] 
siko [8, 9]. Verantwortlich dafür werden insbesondere gesättigte Fettsäuren, der hohe Eisengehalt (Hämeisen), Stickstoffverbindungen (u.a. aus Nitritpökelsalz) sowie zugesetztes Kochsalz in Fleisch und Fleischprodukten gemacht [10].

\section{Hypertonie}

Vegetarier und Veganer sind seltener von Hypertonie betroffen als Mischköstler. Zudem haben sie, insbesondere Veganer, durchschnittlich niedrigere Blutdruckwerte [11, 12]. Eine Ursache dafür liegt in der überdurchschnittlichen Zufuhr von Kalium und Magnesium bei Vegetariern und Veganern beide Mineralstoffe kommen reichlich in Gemüse und Obst vor [13]. Auch pflanzliches Protein wirkt, vermutlich aufgrund seiner Aminosäuren-Zusammensetzung, blutdrucksenkend [14]. Dagegen ist Fleischverzehr meist mit einer erhöhten Zufuhr von Fett (v.a. gesättigte Fettsäuren) und Cholesterin verbunden, was sich blutdrucksteigernd auswirkt [15]. Insbesondere der Konsum von rotem Fleisch und Fleischprodukten ist mit erhöhtem Blutdruck assoziiert [16].

\section{Kardiovaskuläre Erkrankungen}

Das Risiko, an ischämischer Herzkrankheit zu sterben, ist für Vegetarier im Vergleich zu Nicht-Vegetariern um etwa ein Viertel geringer $[17,18]$. Vegetarier und Veganer weisen deutlich seltener die typischen ernährungsbedingten Risikofaktoren für Atherosklerose und Herz-Kreislauf-Erkrankungen auf. Hierzu zählen insbesondere Dyslipidämien wie die Hypercholesterinämie (v.a. LDL-Cholesterin). Da pflanzliche Lebensmittel kein Cholesterin enthalten sowie weniger gesättigte und mehr einfach und mehrfach ungesättigte Fettsäuren liefern, weisen vegetarisch lebende Menschen meist einen deutlich geringeren Gesamt- und LDL-Cholesterinspiegel auf. Bei veganer Ernährung zeigt sich dies noch deutlicher [19]. Auch weitere kardiovaskuläre Risikofaktoren, wie Übergewicht und Adipositas, Diabetes mellitus Typ 2 und Hypertonie kommen bei Vegetariern und Veganern deutlich seltener vor [3].

Für diese positiven Wirkungen sind verschiedene pflanzliche Lebensmittelgruppen verantwortlich. So verringert ein hoher Obstund Gemüseverzehr das Erkrankungsrisiko für Herz-Kreislauf-Krankheiten um 15-35\% $[20,21]$ und der Konsum von Vollkornprodukten das Risiko um etwa 20\%, im Vergleich mit einem niedrigen Verzehr [22]. Besonders protektiv wirkt Nussverzehr: Stehen mindestens 5 Portionen Nüsse pro Woche auf dem Speiseplan, kann das kardiovaskuläre Sterberisiko um bis zu 37\% gesenkt werden [23]. Diese protektiven Wirkungen gehen auf den hohen Gehalt an Vitaminen, Mineralstoffen, Ballaststoffen und sekundären Pflanzenstoffen in pflanzlichen Lebensmitteln zurück, wobei das natürliche Zusammenwirken der einzelnen Stoffe eine entscheidende Rolle spielt.

Studien zeigen, dass Fleischkonsum ein unabhängiger Risikofaktor für die Entstehung von kardiovaskulären Erkrankungen ist [2426 ], ebenso wie ein hoher Verzehr von Eiern ( $\geq 7$ Stück/Woche) das Risiko für Herzinsuffizienz steigert [27].

\section{Krebs}

Bei der Entstehung von Krebs spielt neben weiteren Lebensstilfaktoren (v.a. Rauchen) die Ernährung eine entscheidende Rolle: Schätzungsweise 35\% aller Krebserkrankungen gehen auf Ernährungseinflüsse zurück [28]. So werden bspw. ein geringer Obst- und Gemüseverzehr, eine ballaststoffarme Kost sowie der häufige Konsum von rotem und/ oder verarbeiteten Fleisch als Risikofaktoren für die Krebsentstehung diskutiert [29, 30]. Pflanzenbasierte Ernährungsweisen liefern reichlich Antioxidanzien, sekundäre Pflanzenstoffe und Ballaststoffe, was sich günstig auf das Krebsrisiko auswirkt [28, 30]. Vegetarier und Veganer sind im Durchschnitt besser

\section{Begriffsbestimmung vegetarisch/vegan}

Vegetarisch: kein Verzehr von Nahrungsmitteln, die von toten Tieren stammen, einschließlich daraus hergestellter Erzeugnisse (Fleisch, Wurst, Fisch und andere Meerestiere, gelatinehaltige Gummibärchen u.ä.)

Vegan: Meiden aller von Tieren stammenden Nahrungsmittel, Erzeugnisse und Inhaltsstoffe (Fleisch, Wurst und Fisch; außerdem Milch und Milchprodukte, Eier, Honig; Milchschokolade, Backwaren mit Eiern, mit Gelatine geklärte Getränke [z. B. Wein] usw.; Leder, Wolle, Seide, Federbetten, Reinigungsmittel mit Molke, Kosmetika mit Lanolin u. ä.)

von Lakto-Ovo-Vegetariern 24\% niedriger. Veganer wiesen ein um 16\% niedrigeres Risiko für alle Krebsarten auf und ein 34\% niedrigeres Risiko für Frauen-spezifische Krebsarten [34]. Eine Meta-Analyse von 7 Studien (etwa 125000 Teilnehmer) kam zu dem Ergebnis, dass das Erkrankungsrisiko für Krebs (alle Tumorarten) bei Vegetariern etwa $18 \%$ geringer ist als bei den omnivoren Vergleichsgruppen [35].

\section{Weitere Erkrankungen}

Auch bei Hyperurikämie und Gicht, Divertikulose, Gallen- und Nierensteinen sowie Katarakt wird in den wenigen vorliegenden Studien ein niedrigeres Erkrankungsrisiko für Vegetarier berichtet. Indifferent ist die Studienlage bei Demenzerkrankungen [3].

\section{Vegetarier und Veganer sind seltener betroffen von ernährungsassoziierten Krankheiten wie}

\section{Typ-2-Diabetes, Adipositas, KHK.}

mit diesen gesundheitsfördernden Substanzen versorgt [31, 32]. Fleischkonsum, v.a. von rotem und verarbeitetem Fleisch, erhöht hingegen mit überzeugender Evidenz das Risiko, an Dickdarm- oder Mastdarmkrebs zu erkranken [29, 30, 33].

Die derzeitige Studienlage deutet darauf hin, dass Vegetarier und Veganer ein geringeres Krebsrisiko aufweisen als Fleischesser. In der Adventist Health Study 2 wurde bei Lakto-Ovo-Vegetariern ein geringfügig niedrigeres Gesamt-Krebsrisiko (-7\%) gegenüber Nicht-Vegetariern ermittelt, bei Tumoren des Verdauungstrakts lag das Risiko

\section{Therapie}

Verschiedene Interventionsstudien mit vegetarischen Kostformen zeigten therapeutische Erfolge.

\section{Diabetes Typ 2}

In mehreren Studien konnte durch eine fettarme vegane Ernährung (Dauer 22 und 74 Wochen) im Vergleich zu einer konventionellen Ernährungstherapie bei Typ-2-Diabetikern eine wirkungsvollere Verbesserung der Blutzuckerkontrolle und der Blutfettwerte erreicht werden. Auch die Dosis der medikamentösen Behandlung konnte bei 
der veganen Gruppe nachhaltig reduziert werden [36] (siehe auch zkm 2013; 3: 42).

\begin{abstract}
Hypertonie
Interventionen mit lakto-ovo-vegetarischer Kost (Dauer 6 Wochen) führten sowohl bei normotensiven Personen als auch bei Personen mit leicht erhöhten Blutdruckwerten zu einer Senkung des systolischen Blutdrucks um 5-6 mmHg [37]. Bei 500 Teilnehmern einer 12-tägigen Interventionsstudie bewirkte eine fettarme vegane Kost, in Kombination mit moderater körperlicher Aktivität und Stressmanagement, eine durchschnittliche Blutdrucksenkung von $6 \%$ [38].
\end{abstract}

\section{Kardiovaskuläre Erkrankungen}

Bei 151 Herzpatienten wurden die Auswirkungen einer lakto-vegetarischen Kost sowie einer fettmodifizierten und cholesterinreduzierten Mischkost auf Blutparameter verglichen. Während des stationären Beobachtungszeitraums von 24 Tagen sanken bei der vegetarischen Gruppe im Vergleich zur Kontrollgruppe Gesamtcholesterin (31,1 vs. $16,9 \mathrm{mg} / \mathrm{dl}$ ) und LDL-Cholesterin (27,7 vs. $11,8 \mathrm{mg} / \mathrm{dl}$ ) in signifikant höherem Ausmaß. Die HDL-Cholesterinkonzentration war unverändert [39]. Ein Review über 14 Interventionsstudien ergab, dass sich die Triglyzeridund LDL-Cholesterinkonzentrationen bei

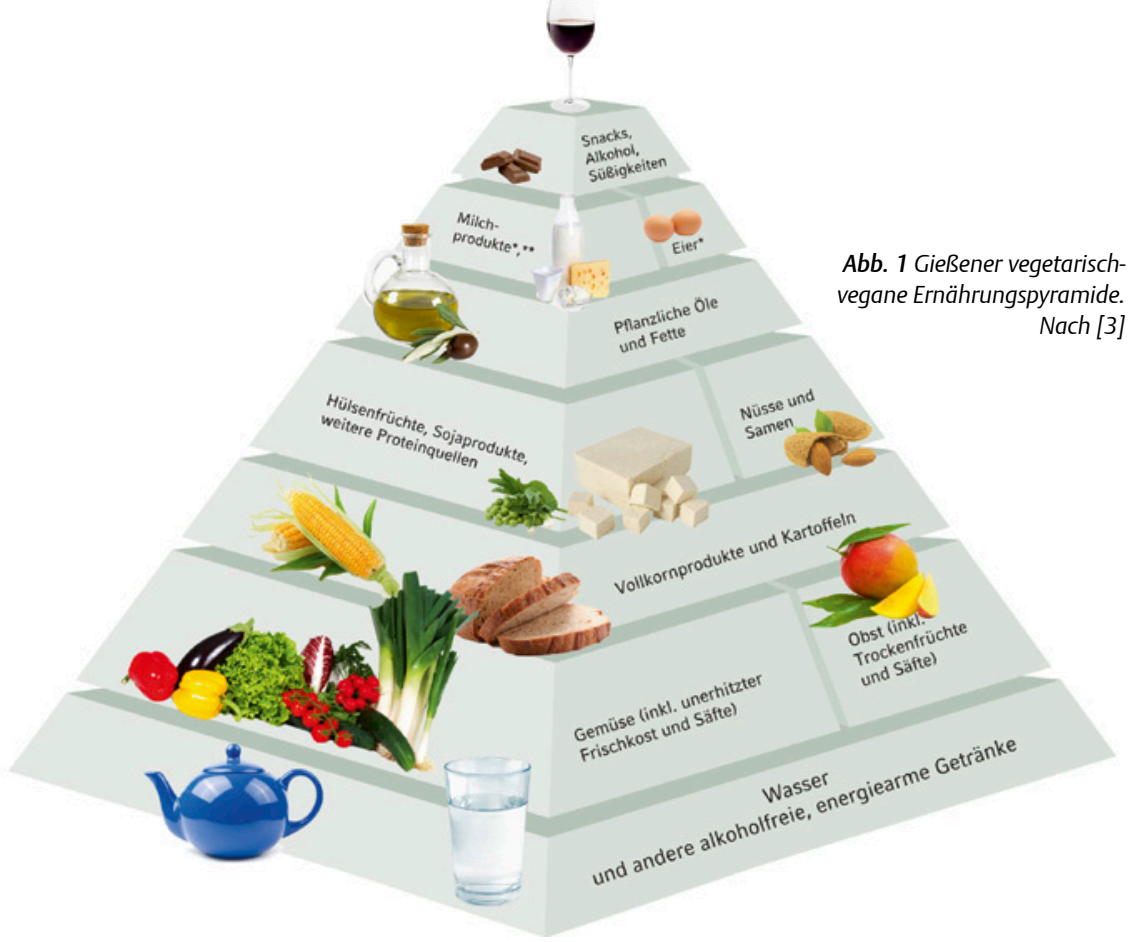

tenstudien. Während zu bestimmten Nährstoffen (z.B. Eisen) und Personengruppen (z.B. Erwachsene) zahlreiche Untersuchungen durchgeführt wurden, besteht bei anderen Nährstoffen und Bevölkerungsgruppen noch erheblicher Forschungsbedarf (z. B. Selen, Omega-3-Fettsäuren; vegetarische und vegane Ernährung bei Schwangeren, Stillenden, Kindern und Sportlern).

Vegetarier und Veganer liegen bei der Zufuhr der Hauptnährstoffe Protein, Fett und Kohlenhydrate deutlich näher an den Empfehlungen der Fachgesellschaften als

\section{Vegetarische Ernährung bietet ein großes}

\section{Potenzial zur Therapie ernährungsassoziierter}

\section{chronischer Krankheiten.}

überwiegend pflanzlichen bzw. lakto-ovovegetarischen Interventionen um durchschnittlich $10-15 \%$ und bei veganen Interventionen um $15-25 \%$ verringerten [40].

\section{Rheumatoide Arthritis}

Bei rheumatoider Arthritis führte die Umstellung auf eine vegetarische oder vegane Kost in verschiedenen Untersuchungen zur Symptomverbesserung [41, 42].

\section{Nährstoffversorgung von Vegetariern und Veganern}

Die vorliegenden Erkenntnisse zur Nährstoffzufuhr und -versorgung bei vegetarischer Ernährung stammen überwiegend aus Querschnitts- und teilweise aus Kohor- die Durchschnittsbevölkerung. Am ehesten erreichen Veganer die Empfehlungen, sowohl relativ (Energie\%) als auch absolut ( $\mathrm{Zu}-$ fuhr in g/d) [3]. Allerdings wurden, insbesondere bei vegan lebenden Frauen, auch teilweise unzureichende Nahrungsenergieund Proteinaufnahmen beobachtet.

Mit vielen Mikronährstoffen sind Vegetarier und Veganer besser versorgt als Mischköstler, insbesondere mit Provitamin $A$ ( $\beta$-Carotin), den Vitaminen $B_{1}, C, E$, Biotin, Pantothensäure und Folat sowie dem Mineralstoff Magnesium, außerdem mit Ballaststoffen und sekundären Pflanzenstoffen [3].

Auf die Zufuhr potenziell kritischer Nährstoffe sollte bei vegetarischen Ernährungsformen jedoch geachtet werden (potenziell kri- tisch bedeutet, dass eine höhere Wahrscheinlichkeit besteht, die empfohlene Zufuhr zu unterschreiten). Hierzu zählen bei lakto-ovovegetarischer Ernährung Eisen, Zink, Omega3-Fettsäuren, Jod und Vitamin $D$, bei veganer Ernährung außerdem Vitamin $B_{12}$, Vitamin $B_{2}$ und Kalzium. Jod und Vitamin D (zwischen Oktober und März) betreffen - unabhängig von der Ernährungsweise - die Gesamtbevölkerung. Auch Eisen und Kalzium sind kritische Nährstoffe in Teilen der Allgemeinbevölkerung.

Häufiger als bei nicht-vegetarischen Vergleichsgruppen wurde eine niedrige Versorgung beobachtet bei [3]:

- Eisen: vegetarisch lebende Kinder, Teenagerinnen und Schwangere; Veganerinnen

- Zink: vegetarisch lebende Teenagerinnen - Vitamin $\mathrm{B}_{12}$ : $>50 \%$ der vegan lebenden Personen; teilweise auch bei Lakto-OvoVegetariern

- Vitamin $\mathrm{B}_{2}$ : lakto-ovo-vegetarisch lebende Teenagerinnen, Veganer

- Kalzium: Veganer (durchschnittliche Zufuhr $40-50 \%$ niedriger als bei Nicht-Vegetariern und Lakto-Ovo-Vegetariern)

- langkettige Omega-3-Fettsäuren (EPA, DHA): Lakto-Ovo-Vegetarier und Veganer. Vegetarier und Veganer sollten daher auf eine ausreichende Zufuhr der potenziell kritischen Nährstoffe achten. Voraussetzung dafür ist eine abwechslungsreiche und gut zusammengestellte Lebensmittelauswahl auf der Basis naturbelassener, möglichst gering verarbeiteter frischer Lebensmittel. Vitamin $\mathrm{B}_{12}$ muss bei veganer Ernährung durch angereicherte Lebensmittel, Supplemente oder Vitamin- $B_{12}$-Zahnpasta - mög- 
licherweise auch in Kombination - aufgenommen werden. Eine Orientierung, auch für die ärztliche Beratung, bietet die Gießener vegetarisch-vegane Ernährungspyramide (Abb. 1). Weitere Unterstützung bieten Lebensmitteltabellen mit Nährstoffangaben (siehe Beispiel Tab. 2).

\section{Weitere Aspekte vegetarischer Ernährungsformen}

Neben dem gesundheitlichen Nutzen haben vegetarische Ernährungsformen zahlreiche weitere positive Auswirkungen. Eine pflanzenbasierte Kost verbraucht in ihrer Erzeugung weniger Energie und Rohstoffe, ist wassersparender, belegt weniger Landfläche und verursacht deutlich weniger Klimagase [3]. Da weniger oder keine sog. Veredelungsverluste auftreten, ist sie ein wichtiger Beitrag zur Sicherung der Welternährung.
Nach Berechnung des Umweltprogramms der Vereinten Nationen (UNEP) entspricht allein die Menge an Getreide, die im Jahr 2050 in die Tierfütterung gehen wird, dem Nahrungsenergiebedarf von 3,5 Mrd. Menschen [43]. Und nicht zuletzt schont eine zunehmende Verbreitung vegetarischer Ernährungsweisen auch die sog. Nutztiere, die in der industrialisierten Landwirtschaft ein alles andere als tiergerechtes Leben fristen. Im Laufe seines Lebens verzehrt jeder Deutsche über 1000 Tiere - Fische und andere Meerestiere nicht eingerechnet [44].

\section{Fazit und Ausblick}

Vegetarische Ernährungsformen, einschließlich der veganen Ernährung, bieten ein großes Potenzial zur Prävention und Therapie ernährungsassoziierter chronischer Krankheiten. Dieses Potenzial wird bisher kaum genutzt. Die Fokussierung auf potenziell kritische Nährstoffe verkennt die gute bis sehr gute Versorgung von Vegetariern und Veganern mit den meisten Nährstoffen. Entsprechend werden pflanzenbasierte Kostformen - trotz gegenteiliger Studienlage - in Ärztekreisen, der Ernährungswissenschaft und den Medien noch immer meist unter dem Aspekt der vermeintlichen Mangelernährung diskutiert. Fachliche Weiterbildung zum Thema vegetarische und vegane Ernährung fördert die ärztliche Kompetenz, hier sachgerecht zu beraten. Patienten und Ratsuchende sollten ermutigt werden, vegetarische Ernährungsformen zu praktizieren.

Interessenkonflikt: Der Autor ist ehrenamtliches Mitglied des Wissenschaftlichen Beirats des Vegetarierbund Deutschland (VEBU).

Online zu finden unter

http://dx.doi.org/10.1055/s-0033-1357231

\section{Zum Weiterlesen}

Leitzmann C, Keller M. Vegetarische Ernährung. 3. Aufl. Stuttgart: Ulmer; 2013

\section{Literatur}

Die Literaturliste finden Sie online unter: www.thieme-connect.de/ejournals/zkm.

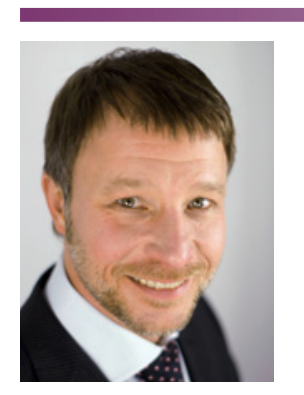

Dr. oec. troph. Markus Keller

Institut für alternative und nachhaltige Ernährung (IFANE)

Niederhofen 11

35428 Langgöns/Gießen

markus.keller@ifane.org

Markus Keller studierte Ökotrophologie an der Universität Gießen, wo er auch promovierte. Im Jahr 2010 gründete er das Institut für alternative und nachhaltige Ernährung (IFANE) in Gießen, das 2013 von der Deutschen UNESCO-Kommission als Offizielles Projekt der UN-Dekade „Bildung für nachhaltige Entwicklung " ausgezeichnet wurde. Schwerpunkte seiner wissenschaftlichen Arbeit sind die Themen Vegetarismus/Veganismus, alternative Ernährungsformen sowie nachhaltige Ernährung; dazu zahlreiche Vorträge, Seminare, TV-, Radio- und Presse-Interviews sowie Veröffentlichungen. Er leitet seit 2011 die Abteilung Wissenschaft und Forschung im Verband für Unabhängige Gesundheitsberatung (UGB) und ist Mitglied des Wissenschaftlichen Beirats des Vegetarierbund Deutschland (VEBU) sowie der Albert Schweitzer Stiftung für unsere Mitwelt. Autor u. a. der Fachbücher „Alternative Ernährungsformen“ und „Vegetarische Ernährung“.

Tab. 2 Kalziumgehalt verschiedener Lebensmittel. Nach [3] 
1 BMELV (Bundesministerium für Ernährung, Landwirtschaft und Verbraucherschutz) und BMG (Bundesministerium für Gesundheit). Gesunde Ernährung und Bewegung [2007]. Im Internet: http://www.gesundheitberlin.de/download/Eckpunkte_Ernaehrung_und_Bewegung.pdf

2 Statistisches Bundesamt. Gesundheitsausgaben im Jahr 2011 bei rund 294 Milliarden Euro. Pressemitteilung Nr. 128 vom 04.04.2013. Im Internet: https://www.destatis.de/DE/PresseService/ Presse/Pressemitteilungen/2013/04/ PD13_128_23611.html

$\overline{3}$ Leitzmann C, Keller M. Vegetarische Ernährung. 3. Aufl. Stuttgart: Ulmer; 2013

$\overline{4}$ Tonstad S, Butler T, Yan R, Fraser GE. Type of vegetarian diet, body weight, and prevalence of type 2 diabetes. Diabetes Care 2009; 32 (5), 791-796

5 Farmer B, Larson BT, Fulgoni VL 3rd, Rainville AJ, Liepa GU. A vegetarian dietary pattern as a nutrient-dense approach to weight management: an analysis of the national health and nutrition examination survey 1999-2004. J Am Diet Assoc 2011; 111 (6), 819-827

$\overline{6}$ Tonstad S, Stewart K, Oda K, Batech M et al. Vegetarian diets and incidence of diabetes in the Adventist Health Study-2. Nutr Metab Cardiovasc Dis 2013; 23 (4), 292-299

7 Hung CJ, Huang PC, Li YH, Lu SC et al. Taiwanese vegetarians have higher insulin sensitivity than omnivores. Br J Nutr 2006; 95 (1), 129-135

$\overline{8}$ Vang A, Singh PN, Lee JW, Haddad EH, Brinegar $\mathrm{CH}$. Meats, processed meats, obesity, weight gain and occurrence of diabetes among adults: findings from Adventists Health Studies. Ann Nutr Metab 2008; 52 (2), 96-104

$\overline{9}$ Aune D, Ursin G, Veierød MB. Meat consumption and the risk of type 2 diabetes: a systematic review and meta-analysis of cohort studies. Diabetologia 2009; 52 (11), 2277-2287

$\overline{10}$ Feskens E], Sluik D, van Woudenbergh GJ. Meat consumption, diabetes, and its complications. Curr Diab Rep 2013; 13 (2), 298-306

$\overline{11}$ Pettersen BJ, Anousheh R, Fan J, Jaceldo-Siegl K, Fraser GE. Vegetarian diets and blood pressure among white subjects: results from the Adventist Health Study-2 (AHS-2). Public Health Nutr 2012; 10, 1-8

$\overline{12}$ Appleby PN, Davey GK, Key T]. Hypertension and blood pressure among meat eaters, fish eaters, vegetarians and vegans in EPIC-Oxford. Public Health Nutr 2002; 5 (5), 645-654

$\overline{13}$ Berkow SE, Barnard ND. Blood pressure regulation and vegetarian diets. Nutr Rev 2005; 63 (1), 1-8

$\overline{14}$ Altorf-van der Kuil W, Engberink MF, Brink EJ, van Baak MA et al. Dietary protein and blood pressure: a systematic review. PLoS One 2010; 5 (8), e12102

$\overline{15}$ Miller ER 3rd, Erlinger TP, Appel Ll. The effects of macronutrients on blood pressure and lipids: an overview of the DASH and OmniHeart trials. Curr Atheroscler Rep 2006; 8 (6), 460-465 $\overline{16}$ Steffen LM, Kroenke CH, Yu X, Peretra MA et al. Associations of plant food, diary product, and meat intakes with 15-y incidence of elevated blood pressure in young black and white adults: the Coronary Artery Risk Development in Young Adults (CARDA) study. Am J Clin Nutr 2005; 82 (6), 1169-1177

$\overline{17}$ Key TJ, Fraser GE, Thorogood M, Appleby PN et al. Mortality in vegetarians and nonvegetarians: detailed findings from a collaborative analysis of 5 prospective studies. Am J Clin Nutr 1999; 70 (3 Suppl), 516S-524S

$\overline{18}$ Huang T, Yang B, Zheng J, Li G, Wahlqvist ML, Li D. Cardiovascular disease mortality and cancer incidence in vegetarians: a meta-analysis and systematic review. Ann Nutr Metab 2012; 60 (4), 233-240

$\overline{19}$ Key TJ, Appleby PN. Vegetarianism, coronary risk factors and coronary heart disease. In: Sabate J, ed. Vegetarian nutrition. Boca Raton: CRC Press; 2001: 33-54

$\overline{20}$ Dauchet L, Amouyel P, Hercberg S, Dallongeville J. Fruit and vegetable consumption and risk of coronary heart disease: a meta-analysis of cohort studies. J Nutr 2006; 136 (10), 2588-2593

$\overline{21}$ Ströhle A, Waldmann, Wolter M, Hahn A. Vegetarische Ernährung: Präventives Potenzial und mögliche Risiken. Teil 1: Lebensmittel pflanzlicher Herkunft. Wien Klin Wochenschr 2006; 118 (23-24), 728-737

$\overline{22}$ Mellen PB, Walsh TF, Herrington DM. Whole grain intake and cardiovascular disease: a metaanalysis. Nutr Metab Cardiovasc Dis 2008; 18 (4), 283-290

$\overline{23}$ Kelly JH Jr, Sabaté J. Nuts and coronary heart disease: an epidemiological perspective. $\mathrm{Br} J$ Nutr 2006; 96 Suppl 2, S61-S67

$\overline{24}$ Kontogianni MD, Panagiotakos DB, Pitsavos C, Chrysohoou C, Stefanadis C. Relationship between meat intake and the development of acute coronary syndromes: the CARDIO2000 case-control study. Eur J Clin Nutr 2008; 62 (2), 171-177

$\overline{25}$ Sinha R, Cross AJ, Graubard BI, Leitzmann MF, Schatzkin A. Meat intake and mortality: a prospective study of over half a million people. Arch Intern Med 2009; 169 (6), 562-571

$\overline{26}$ Pan A, Sun Q, Bernstein AM, Schulze MB et al. Red meat consumption and mortality: results from 2 prospective cohort studies. Arch Intern Med 2012; 172 (7), 555-563

$\overline{27}$ Djoussé L, Gaziano JM. Egg consumption and risk of heart failure in the Physicians'Health Study. Circulation 2008; 117 (4), 512-516

$\overline{28}$ Leitzmann C, Müller C, Michel P, Brehme U et al. Ernährung in Prävention und Therapie. 3. Aufl. Stuttgart: Hippokrates; 2009: 385, 390-393

$\overline{29}$ Deutsche Gesellschaft für Ernährung, Hrsg. Ernährungsbericht. Bonn; 2008

$\overline{30}$ World Cancer Research Fund, American Institute for Cancer Research, ed. Food, nutrition, physical activity, and the prevention of cancer: a global perspective. Washington; 2007 $\overline{31}$ Haldar S, Rowl and IR, Barnett YA , Bradbury I et al. Influence of habitual diet on antioxidant status: a study in a population of vegetarians and omnivores. Eur J Clin Nutr 2007; 61 (8), 1011-1022

$\overline{32}$ Peeters PH, Slimani N, van der Schouw YT, Grace PB et al. Variations in plasma phytoestrogen concentrations in European adults. J Nutr 2007; 137 (5), 1294-1300

$\overline{33}$ Chan DS, Lau R, Aune D, Vieira R et al. Red and processed meat and colorectal cancer incidence: meta-analysis of prospective studies. PLoS One 2011; 6 (6), e20456

$\overline{34}$ Tantamango-Bartley Y, Jaceldo-Siegl K, Fan J, Fraser $\mathrm{G}$. Vegetarian diets and the incidence of cancer in a low-risk population. Cancer Epidemiol Biomarkers Prev 2013; 22 (2), 286-294

$\overline{35}$ Huang T, Yang B, Zheng J, Li G, Wahlqvist ML, Li D. Cardiovascular disease mortality and cancer incidence in vegetarians: a meta-analysis and systematic review. Ann Nutr Metab 2012; 60 (4), 233-240

$\overline{36}$ Trapp CB, Barnard ND. Usefulness of vegetarian and vegan diets for treating type 2 diabetes. Curr Diab Rep 2010; 10 (2), 152-158

$\overline{37}$ Berkow SE, Barnard ND. Blood pressure regulation and vegetarian diets. Nutr Rev 2005; 63 (1), $1-8$

$\overline{38}$ McDougall J, Litzau K, Haver E, Saunders V, Spiller GA. Rapid reduction of serum cholesterol and blood pressure by a twelve-day, very low fat, strictly vegetarian diet. J Am Coll Nutr 1995; 14 (5), 491-496

$\overline{39}$ Brestrich M, Claus J, Blümchen G. Die lactovegetabile Diät: Einfluss auf das Verhalten von Körpergewicht, Lipidstatus, das Fibrinogen und Lipoprotein (a) bei Herzkreislaufkranken während einer stationären Rehabilitationsmaßnahme. Z Kardiol 1996; 85 (6), 418-427

$\overline{40}$ Ferdowsian HR, Barnard ND. Effects of plantbased diets on plasma lipids. Am J Cardiol 2009; 104 (7), 947-956

$\overline{41}$ Müller H, de Toledo FW, Resch KL. Fasting followed by vegetarian diet in patients with rheumatoid arthritis: a systematic review. Scand J Rheumatol 2001; 30 (1), 1-10

$\overline{42}$ McDougall J, Bruce B, Spiller G, Westerdahl J, McDougall M. Effects of a very low-fat, vegan diet in subjects with rheumatoid arthritis. J Altern Complement Med 2002; 8 (1), 71-75

$\overline{43}$ United Nations Environment Programme. The environmental food crisis. Nairobi; 2009: 27

$\overline{44}$ Vegetarierbund Deutschland. Vegetarierbund berechnet: 1094 Tiere verspeist jeder Deutsche [2009]. Im Internet: https://vebu.de/aktuelles/ pressemitteilungen/422-zum-morgigen-welt-vegetariertag-vegetarierbund-berechnet-1094-tiere-verspeist-jeder-deutsche

$\overline{45}$ Keller M, Leitzmann C. Vegetarische Ernährung. Eine Ernährungsweise mit Zukunft. Spiegel der Forschung 2011; 28 (1), 20-30 